\title{
Potential Cardiotoxic Effects of Remdesivir on Cardiovascular System: A Literature Review
}

\author{
Maryam Nabati ${ }^{1}\left[\right.$. Homa Parsaee ${ }^{2}$
}

Received: 14 August 2021 / Accepted: 7 October 2021 / Published online: 13 October 2021

(c) The Author(s), under exclusive licence to Springer Science+Business Media, LLC, part of Springer Nature 2021

\begin{abstract}
Corona disease 2019 (COVID-19) pandemic continues to spread around the world with no efficacious treatment. Intravenous remdesivir is the only authorized drug for treatment of COVID-19 disease under an Emergency Use Authorization. Remdesivir is a 1'-cyano-substituted adenosine nucleotide prodrug which inhibits viral RNA synthesis. This metabolite is an adenosine analog but with a significantly longer half-life than adenosine. Adenosine is a powerful vasodilator that can cause profound hypotension which is followed by the compensatory release of catecholamines. It can also shorten atrial action potential and refractoriness and lead to atrial fibrillation (AF). These effects may also occur in ventricular cells and predispose patients to ventricular fibrillation. Remdesivir can also induce significant cytotoxic effects in cardiomyocytes that is considerably worse than chloroquine cardiotoxic effects. Remdesivir-induced cardiotoxicity is due to its binding to human mitochondrial RNA polymerase. On the other hand, remdesivir can increase field potential duration with decreased $\mathrm{Na}^{+}$peak amplitudes and spontaneous beating rates in a dose-dependent manner that might induce prolonged QT interval and torsade de point. There are some reports of sinus bradycardia, hypotension, T-wave abnormalities, AF, and a prolonged QT interval and few cases of cardiac arrest and complete heat block following remdesivir infusion. It seems remdesivir have some cardiotoxic and proarrhythmic effects that are especially more pronounced in patients with previous cardiovascular diseases. The current safety profile of remdesivir is still not completely known and further prospective clinical trials are needed to assess its safety profile and potential adverse cardiovascular effects.
\end{abstract}

Keywords Corona virus $\cdot$ COVID-19 $\cdot$ Remdesivir $\cdot$ Bradycardia $\cdot$ QTC prolongation $\cdot$ Cardiovascular

\section{Introduction}

Corona disease 2019 (COVID-19) is an infectious disease which continues to spread around the world with no efficacious treatment [1]. It has become a pandemic disease that has manifested itself as the most important health catastrophe of the century. Several medications including chloroquine/hydroxychloroquine, favipiravir, lopinavir/ritonavir,

Handling Editor: Y. James Kang.

Maryam Nabati

Dr.mr.nabati@gmail.com

1 Department of Cardiology, Faculty of Medicine, Cardiovascular Research Center, Fatemeh Zahra Teaching Hospital, Mazandaran University of Medical Sciences, Artesh Boulevard, Sari, Iran

2 Student Research Committee, Faculty of Medicine, Iran University of Medical Sciences, Tehran, Iran azithromycin, and remdesivir have been used in clinical trials and have shown some beneficial effects [2]. Among these drugs, intravenous remdesivir is the only authorized drug for treatment of COVID-19 disease under an Emergency Use Authorization [3]. It is an investigational antiviral drug which was initially used in patients with the Ebola virus [4]. Remdesivir is a 1'-cyano-substituted adenosine nucleotide prodrug which inhibits viral RNA synthesis [2]. Indeed, it works as an adenosine analog by incorporating itself into viral RNA. This leads to premature chain termination and inhibition of viral replication [1]. COVID-19-infected patients with underlying cardiovascular disease seem to have an adverse outcome. Furthermore, a decreased potassium level occurs commonly in patients with COVID-19, which may lead to electrocardiographic changes such as prolonged QT interval and can increase the risk of adverse medical side effects [5]. Therefore, understanding the cardiovascular risks of potential medications is of particular importance. Given that remdesivir is the most important drug in the treatment 
of patients admitted with COVID-19, we decided to perform a literature review about its potential side effects on cardiovascular system.

\section{Mechanism}

Remdesivir molecule is similar to adenosine. Its active form is nucleoside triphosphate remdesivir and remdesivir (GS441524) which is accessible in cytoplasm without ability to diffuse back to extracellular matrix. The active form is used by cell viral RNA-dependent RNA polymerase instead of the adenosine during replication of viral genome. This process can interrupt viral replication which is irreversible and unable to repair. The FDA Emergency Use Authorization recommends a loading dose of $200 \mathrm{mg}(5 \mathrm{mg} / \mathrm{kg})$ intravenous infusion once on the first day and $100 \mathrm{mg}$ for the following days (for 5 to 10 days) [6, 7]. Its initiation is generally recommended when oxygen saturations fall below $94 \%$ [1].

\section{Adverse Non-cardiac Side Effects}

The most common adverse side effects of remdesivir are anemia, infusion site reactions, increased liver enzymes, cutaneous rash, kidney injuries, and hypotension [1]. Also, worsening or respiratory failure, pyrexia, hyperglycemia, diarrhea, multiple organ failure, hypoalbuminemia, hypokalemia, hypernatremia, thrombocytopenia, and septic shock occurred more frequently in remdesivir than control group [6].

\section{Literature Review on Cardiac Side Effects}

There are few studies focused on remdesivir and its adverse effects on the cardiovascular system.

Table 1 shows selected published studies that reported adverse effects of remdesivir on cardiovascular system. In 2020, Grein et al. conducted a study on 61 hospitalized patients with COVID-19 infection who had an oxygen saturation of $94 \%$ or less. Patients received intravenous remdesivir for 10 days and were followed up for 28 days. Any adverse event that lasted more than a day was considered a side effect. Besides more common non-cardiac adverse effects, four patients experienced hypotension and three patients revealed an atrial fibrillation (AF) rhythm. Adverse events were more prevalent in patients undergoing invasive ventilation. However, interpretation of these findings is not simply possible. It is due to small sample size, short duration of follow-up, some missing data, and the absence of a randomized control group [8]. In 2020, Gupta et al. evaluated two patients diagnosed with coronavirus infection who had

Table 1 Selected published studies that reported adverse effects of remdesivir on cardiovascular system

\begin{tabular}{|c|c|c|c|c|c|}
\hline Authors & Study design & Year & $\begin{array}{l}\text { The number of COVID-19 } \\
\text { patients with an adverse } \\
\text { event }\end{array}$ & Follow-up time & Side effects \\
\hline Grein et al & Cohort & 2020 & 7 & 28 days & Hypotension, AF rhythm \\
\hline Gupta et al & Case presentation & 2020 & 2 & During hospitalization & $\begin{array}{l}\text { Bradycardia, Prolonged QT } \\
\text { interval, T-wave abnor- } \\
\text { mality }\end{array}$ \\
\hline Michaud et al & Pharmacological study & 2021 & Not applicable & Not applicable & $\begin{array}{l}\text { Prolonged QT interval, } \\
\text { Torsade de point }\end{array}$ \\
\hline Mulangu et al & $\begin{array}{l}\text { A randomized, controlled } \\
\text { trial }\end{array}$ & 2019 & 1 & During hospitalization & Hypotension, cardiac arrest \\
\hline Wang et al & $\begin{array}{l}\text { A randomized, double-blind, } \\
\text { placebo-controlled, multi- } \\
\text { center trial }\end{array}$ & 2020 & 1 & During hospitalization & Cardiac arrest \\
\hline Gubitosa et al & Case presentation & 2020 & 1 & During hospitalization & $\begin{array}{l}\text { Sinus bradycardia and QRS } \\
\text { widening }\end{array}$ \\
\hline Bistrovic et al & Pilot study & 2021 & 10 & During hospitalization & $\begin{array}{l}\text { T-wave rightward axis } \\
\text { deviation }\end{array}$ \\
\hline Rafaniello et al & Meta-analysis & 2021 & Twofold increased risk & During hospitalization & Adverse cardiac event \\
\hline Sanchez-Codez et al & Case presentation & 2021 & 1 & During hospitalization & Sinus bradycardia \\
\hline Selvaraj et al & Case presentation & 2021 & 1 & During hospitalization & Complete heart block \\
\hline Barkas et al & Case presentation & 2021 & 1 & During hospitalization & Sinus bradycardia \\
\hline Chow et al & Case presentation & 2021 & 1 & During hospitalization & Sinus bradycardia \\
\hline
\end{tabular}

$A F$ atrial fibrillation 
experienced worsening respiratory condition and received antibiotics including ceftriaxone and azithromycin, corticosteroids, and remdesivir. First patient was a 26-year-old African American female. She experienced sinus bradycardia with a heart rate of $40-50$ beats per minute (bpm), prolonged corrected QT interval of $555 \mathrm{~ms}$, and T-wave abnormality after receiving third dose of remdesivir. After discontinuation of remdesivir, all changes were returned to normal. Her serum potassium was within the normal range, indicating potassium did not have a significant role. Azithromycin discontinued shortly before remdesivir initiation that made it as a possible contributing factor for prolongation of the QT interval. Second patient was a 77-year-old Caucasian female who developed sinus bradycardia with a heart rate of $48 \mathrm{bpm}$ on day three after remdesivir administration. The patient's heart rate returned to baseline on the following days after discontinuation of remdesivir. Sinus bradycardia had been seen in both of these patients within 20-40 min of infusion time (at peak dose) [1]. In 2021, Michaud et al. performed a study about the risk of drug-induced long-QT syndrome associated with COVID-19 medications by comparing them with 23 well-known torsadogenic and 10 low torsadogenicrisk compounds. Pharmacokinetic and pharmacodynamic data were computed to measure affinity for blocking the rapid component of the delayed rectifier cardiac potassium current and tendency toward a prolonged cardiac repolarization and torsade de pointes. The results of this study showed that remdesivir can significantly increase the risk of QT prolongation and torsade de point, especially with an elevated plasma concentration following intravenous administration. Therefore, they recommended close monitoring on the QT interval during remdesivir therapy [4]. In 2020, Davies et al. performed a systematic benefit-risk assessment to determine the overall benefit-risk of the use of remdesivir in comparison with placebo or other therapies. They suggested that remdesivir may have a favorable benefit-risk profile in comparison with placebo in severe COVID-19 infection. They pointed out that cardiovascular outcomes including hypotension and arrhythmias were documented with use of remdesivir [9]. Hypotension was reported in one patient with Ebola virus during remdesivir infusion that was followed by a cardiac arrest, although authors declare that this death might also be due to underlying fulminant Ebola virus [10]. Wang et al. did a randomized, double-blind, placebo-controlled, multicenter trial on 237 hospitalized patients with severe COVID-19 who were enrolled and randomly assigned to a treatment group (158 to remdesivir and 79 to placebo). They did not find any statistically significant clinical benefits with remdesivir. However, there was a reduction in time to clinical improvement in those treated earlier with remdesivir compared with placebo. One patient in remdesivir group suffered from a cardiac arrest compared with no case in placebo group [11]. Gubitosa et al. reported a 54-year-old female diagnosed with corona virus who had experienced a worsening dyspnea, fever, and chills for more than 2-week duration. Other vital signs were within the normal ranges and oxygen saturation on the room air was $95 \%$. The baseline heart rate was $60-70 \mathrm{bpm}$. The patient's past medical history revealed an unknown origin left bundle branch block, hypertension, and lymphoma. Remdesivir was initiated 2 days after admission. Patient developed a sinus bradycardia with a heart rate of $38 \mathrm{bpm}$ with $24 \mathrm{~h}$. Other vital signs and cardiac biomarkers were within normal limits. Her heart rate decreased to $34 \mathrm{bpm}$ and she complained of dizziness and chest discomfort and developed hypotension in the next two days. An electrocardiogram was taken which revealed a QRS widening of $170 \mathrm{~ms}$. Remdesivir was discontinued and atropine was administrated. The patient's heart rate reverted to the baseline and QRS complex decreased to $168 \mathrm{~ms}$ and her related symptoms improved within the following days [12]. In 2021, Bistrovic et al. performed a pilot study on 14 consecutive patients with severe COVID-19 treated by remdesivir and dexamethasone. An electrocardiogram was recorded before and $2 \mathrm{~h}$ after infusion of remdesivir. A total of 10 patients experienced a statistically significant effect of remdesivir on the $\mathrm{T}$-wave axis deviation including rightward axis deviation [13]. The T-axis deviation is a powerful risk factor of sudden cardiac death, cardiac death, and non-fatal cardiac events in aged individuals [14]. In 2021, Rafaniello et al. analyzed all Individual Case Safety Reports (ICSRs) collected in the Eudra Vigilance database focusing on cardiac adverse events that 1375 ICSRs related to remdesivir were retrieved. The most commonly reported adverse events were hepatic/hepatobiliary disorders (19.4\%), kidney and urinary involvements $(11.1 \%)$, and cardiac events (8.4\%). Among 221 cardiac ICSRs, 69 patients experienced a fatal outcome. Data analysis revealed a twofold increased risk of an adverse cardiac event associated with remdesivir in comparison with hydroxychloroquine and azithromycin. The result of this study showed that remdesivir might increase the risk of adverse cardiac events [15]. Sanchez-Codez et al. reported a 13-year-old boy with a history of bronchial asthma who was admitted with severe bilateral pneumonia and hypoxemia by COVID-19 with a baseline heart rate of 80-90 bpm. He received oxygen therapy, dexamethasone, ceftriaxone, and remdesivir (a loading dose of $200 \mathrm{mg} / \mathrm{day}$ that was followed by $100 \mathrm{mg} /$ day). He developed an asymptomatic and nonhemodynamically significant sinus bradycardia (a heart rate of $40 \mathrm{bpm}$ ) following the third dose of remdesivir. Remdesivir infusion was stopped which consequently resulted in normalization of the heart rate in the following day. Cardiac biomarker levels and echocardiographic variables were within the normal range. They claimed that bradycardia in this patient occurred due to the fact that remdesivir was an adenosine analog with a tendency to block the atrioventricular (AV) node [16]. Selvaraj 
et al. reported a 72-year-old male with a previous history of $\mathrm{AF}$, right bundle branch block, and pulmonary cancer who was hospitalized for COVID-19. Intravenous remdesivir and dexamethasone were initiated. He developed an AF with ventricular rates between 30 and $40 \mathrm{bpm}$ following the first day of initiating remdesivir. On the fifth day of remdesivir treatment, patient developed a complete AV block. Patient remained asymptomatic and received full remdesivir regimen under close cardiac rhythm monitoring. The AV block resolved spontaneously within the next $48 \mathrm{~h}$ [17]. Barkas et al. reported a 36-year-old male hospitalized with severe COVID-19 symptoms, including prolonged fever, hypoxemia, myalgia, and hemoptysis. An electrocardiogram revealed a normal sinus rhythm with a heart rate of $92 \mathrm{bpm}$. He received oxygen, corticosteroid, remdesivir, and antibiotic therapy. An asymptomatic sinus bradycardia with a heart rate of $39 \mathrm{bpm}$ developed on third day. Secondary causes of sinus bradycardia were ruled out based on laboratory and echocardiographic findings. The rhythm reverted to normal 9 days after discontinuation of remdesivir [18]. Chow et al. described a 16-year-old obese male with COVID-19 disease hospitalized with fever, respiratory distress, dry cough, headache, and gastrointestinal symptoms. The patient's baseline heart rate was more than $90 \mathrm{bpm}$. He had mild hypoxia and the treating physicians decided that the patient should not receive corticosteroids. Remdesivir treatment was initiated on the second day of hospitalization. This was followed by a decrease in heart rate ranged between 40 and $60 \mathrm{bpm}$ over the next 4 days. Patient was not hypotensive and echocardiography revealed normal left ventricular size and systolic function. Two days after discontinuation of remdesivir, the patient's heart rate reverted to the baseline value [19].

\section{Discussion}

Remdesivir is a nucleotide analog prodrug that is activated to a nucleoside triphosphate metabolite (GS-443902). This metabolite is an adenosine analog but with a significantly longer half-life than adenosine with an elimination half-life of $11 \mathrm{~h}$. Also, there is another dephosphorylated nucleoside metabolite (GS-441524) that has a half-life of 20-25 h [13, 17]. Adenosine has been documented to have antiarrhythmic effects in AV re-entrant tachycardias by inducing transient AV nodal block. However, it may be proarrhythmic in patients with structural heart disease [13]. Adenosine is a powerful vasodilator that can cause profound hypotension which is followed by the compensatory release of catecholamines. These events can lead to ventricular tachycardia or even degenerate into ventricular fibrillation (VF). It can also shorten atrial action potential and refractoriness and lead to AF. These effects may also occur in ventricular cells and predispose patients to VF. On the other hand, adenosine may lead to VF by inducing spatially and temporally inhomogeneity in ventricular refractoriness [20]. Remdesivir is structurally similar with adenosine, but with a longer half-life. Therefore, its adverse cardiovascular effects are not unexpected.

Choi et al. generated human pluripotent stem cell-derived cardiomyocytes (hPSC-CMs) from human embryonic stem cells and human-induced pluripotent stem cells to determine the potential cardiovascular risk associated with remdesivir treatment in COVID-19-infected hPSC-CMs. This study revealed that remdesivir can induce significant cytotoxic effects in cardiomyocytes that is considerably worse than chloroquine cardiotoxic effects [3]. Remdesivir-induced cardiotoxicity is due to its binding to human mitochondrial RNA polymerase [16]. Furthermore, longer duration of remdesivir treatment $(48 \mathrm{~h})$ considerably decreased viability of $\mathrm{CMs}$ in comparison with shorter treatment time $(24 \mathrm{~h})$ that was suggestive of a potential accumulative toxicity with remdesivir treatment. Remdesivir-induced cardiotoxicity in hPSC-CMs occurred with 50\% cytotoxic concentration $\left(\mathrm{CC}_{50}\right)$ values close to its estimated peak plasma concentration $\left(C_{\max } 9 \mu \mathrm{M}\right)$ as have been reported in the literature. On the other hand, remdesivir dose dependently increased field potential duration with decreased $\mathrm{Na}^{+}$peak amplitudes and spontaneous beating rates that lead to complete cessation of spontaneous beating at higher concentrations. These findings showed that overdose or drug accumulation might induce serious adverse cardiac effects. This means that a remdesivir concentrations at a level higher than the estimated peak plasma concentration $\left(C_{\max } 9 \mu \mathrm{M}\right)$ is associated with the potential risk of QT prolongation. Therefore, close monitoring of the electrocardiogram and QT interval is advisable during remdesivir medication, especially in patients with structural heart disease or severe COVID-19-infected individuals [3].

\section{Conclusion}

Some patients with COVID-19 infection on remdesivir may develop sinus bradycardia, hypotension, T-wave abnormalities, AF, and a prolonged QT interval. Also, few cases of cardiac arrest and complete heat block following remdesivir infusion have been reported. It seems remdesivir have some cardiotoxic and proarrhythmic effects that are especially more pronounced in patients with previous cardiovascular diseases. Continuous cardiac rhythm monitoring is recommended in patients undergoing remdesivir treatment. It is especially advisable in individuals with any known cardiac diseases or electrolyte disturbances. Furthermore, we should be more cautious about additive cardiovascular adverse effects when other drug classes are used in addition 
to remdesivir treatment. It is due to the fact that their combination may increase the risk of potential fatal ventricular arrhythmias or cardiac arrest. The current safety profile of remdesivir is still not completely known. Further prospective clinical trials can assist us to know more about its safety profile and potential adverse cardiovascular effects.

\section{References}

1. Gupta, A. K., Parker, B. M., Priyadarshi, V., \& Parker, J. (2020). Cardiac adverse events with remdesivir in COVID-19 infection. Cureus, 12(10), e11132.

2. Aggarwal, G., Henry, B. M., Aggarwal, S., \& Bangalore, S. (2020). Cardiovascular safety of potential drugs for the treatment of coronavirus disease 2019. The American Journal of Cardiology, 128, 147-150.

3. Choi, S. W., Shin, J. S., Park, S.-J., Jung, E., Park, Y.-G., Lee, J., et al. (2020). Antiviral activity and safety of remdesivir against SARS-CoV-2 infection in human pluripotent stem cell-derived cardiomyocytes. Antiviral Research, 184, 104955.

4. Michaud, V., Dow, P., Al Rihani, S. B., Deodhar, M., Arwood, M., Cicali, B., et al. (2021). Risk Assessment of drug-induced long QT syndrome for some COVID-19 repurposed drugs. Clinical and Translational Science, 14(1), 20-28.

5. Alfano, G., Ferrari, A., Fontana, F., Perrone, R., Mori, G., Ascione, E., et al. (2021). Hypokalemia in patients with COVID19. Clinical and Experimental Nephrology, 25(4), 401-409.

6. Badgujar, K. C., Ram, A. H., Zanznay, R., Kadam, H., \& Badgujar, V. C. (2020). Remdesivir for COVID-19: A review of pharmacology, mechanism of action, in-vitro activity and clinical use based on available case studies. Journal of Drug Delivery and Therapeutics, 10(4-s), 264-270.

7. Humeniuk, R., Mathias, A., Cao, H., Osinusi, A., Shen, G., Chng, E., et al. (2020). Safety, tolerability, and pharmacokinetics of remdesivir, an antiviral for treatment of COVID-19, in healthy subjects. Clinical and Translational Science, 13(5), 896-906.

8. Grein, J., Ohmagari, N., Shin, D., Diaz, G., Asperges, E., Castagna, A., et al. (2020). Compassionate use of remdesivir for patients with severe COVID-19. New England Journal of Medicine, 382(24), 2327-2336.

9. Davies, M., Osborne, V., Lane, S., Roy, D., Dhanda, S., Evans, A., et al. (2020). Remdesivir in treatment of COVID-19: A systematic benefit-risk assessment. Drug Safety, 43, 645-656.

10. Mulangu, S., Dodd, L. E., Davey, R. T., Jr., Tshiani Mbaya, O., Proschan, M., Mukadi, D., et al. (2019). A randomized, controlled trial of Ebola virus disease therapeutics. New England Journal of Medicine, 381(24), 2293-2303.
11. Wang, Y., Zhang, D., Du, G., Du, R., Zhao, J., Jin, Y., et al. (2020). Remdesivir in adults with severe COVID-19: A randomised, double-blind, placebo-controlled, multicentre trial. The Lancet, 395(10236), 1569-1578.

12. Gubitosa, J. C., Kakar, P., Gerula, C., Nossa, H., Finkel, D., Wong, K., et al. (2020). Marked sinus bradycardia associated with remdesivir in COVID-19: A case and literature review. Case Reports, 2(14), 2260-2264.

13. Bistrovic, P., \& Lucijanic, M. (2021). Remdesivir might induce changes in electrocardiogram beyond bradycardia in patients with Coronavirus disease 2019 (COVID-19) - The pilot study. Journal of Medical Virology, 93(10), 5724-5725.

14. Rautaharju, P. M., Nelson, J. C., Kronmal, R. A., Zhang, Z.-M., Robbins, J., Gottdiener, J. S., et al. (2001). Usefulness of T-axis deviation as an independent risk indicator for incident cardiac events in older men and women free from coronary heart disease (the Cardiovascular Health Study). The American Journal of Cardiology, 88(2), 118-123.

15. Rafaniello, C., Ferrajolo, C., Sullo, M. G., Gaio, M., Zinzi, A., Scavone, C., et al. (2021). Cardiac events potentially associated to remdesivir: An analysis from the European Spontaneous Adverse Event Reporting System. Pharmaceuticals, 14(7), 611.

16. Sanchez-Codez, M. I., Rodriguez-Gonzalez, M., \& GutierrezRosa, I. (2021). Severe sinus bradycardia associated with remdesivir in a child with severe SARS-CoV-2 infection. European Journal of Pediatrics, 180(5), 1627.

17. Selvaraj, V., Bavishi, C., Patel, S., \& Dapaah-Afriyie, K. (2021). Complete heart block associated with Remdesivir in COVID19: A case report. European Heart Journal Case Reports, 5(7), ytab200.

18. Barkas, F., Styla, C.-P., Bechlioulis, A., Milionis, H., \& Liberopoulos, E. (2021). Sinus bradycardia associated with remdesivir treatment in COVID-19: A case report and literature review. Journal of Cardiovascular Development and Disease, 8(2), 18.

19. Chow, E. J., Maust, B., Kazmier, K. M., \& Stokes, C. (2021). Sinus bradycardia in a pediatric patient treated with remdesivir for acute coronavirus disease 2019: A case report and a review of the literature. Journal of the Pediatric Infectious Diseases Society. https://doi.org/10.1093/jpids/piab029

20. Parham, W. A., Mehdirad, A. A., Biermann, K. M., \& Fredman, C. S. (2001). Case report: Adenosine induced ventricular fibrillation in a patient with stable ventricular tachycardia. Journal of Interventional Cardiac Electrophysiology, 5(1), 71-74.

Publisher's Note Springer Nature remains neutral with regard to jurisdictional claims in published maps and institutional affiliations. 